\title{
SODIUM ALGINATE/GELATIN MICROBEADS-INTERCALATED WITH KAOLIN NANOCLAY FOR EMERGING DRUG DELIVERY IN WILSON'S DISEASE
}

\author{
O. SREEKANTH REDDY1, M. C. S. SUBHA ${ }^{1}{ }^{*}$, T. JITHENDRA ${ }^{1}$, C. MADHAVI ${ }^{2}$, K. CHOWDOJI RAO ${ }^{2}$, B. MALLIKARJUNA ${ }^{3}$ \\ ${ }^{1}$ Department of Chemistry, Sri Krishnadevaraya University, Ananthapuramu 515003, India, ${ }^{2}$ Department of Polymer Science and \\ Technology, Sri Krishnadevaraya University, Ananthapuramu 515003, India, ${ }^{3}$ Department of Chemistry, Government College (A), AKN \\ University, Rajahmundry, India \\ Email: sreekanthchem7@gmail.com
}

Received: 22 May 2019, Revised and Accepted: 04 Jul 2019

\section{ABSTRACT}

Objective: The aim of the present study was to fabricate and evaluate the drug release studies using Sodium Alginate (SA) and Gelatin (GE) microbeads intercalated with Kaolin (KA) nanoclay for sustained release of D-Penicillamine (D-PA).

Methods: Sodium alginate/gelatin/Kaolin blend microbeads were prepared by an extrusion method by using glutaraldehyde (GA) as a crosslinker. The obtained microbeads were characterized by Fourier transform infrared (FTIR) spectroscopy, scanning electron microscopy (SEM) and X-ray diffraction (XRD). Drug release kinetics of the microbeads was investigated in simulated intestinal fluid ( $\mathrm{pH} 7.4$ ) at $37{ }^{\circ} \mathrm{C}$.

Results: Microbeads formation was confirmed by FTIR spectroscopy. X-RD reveals that the KA should be intercalated with the drug and also it confirms the molecular level dispersion of D-Penicillamine into microbeads. Scanning Electron Microscopy (SEM) studies reveal that the beads were in spherical shape with some wrinkled depressions on the surface. The in vitro release study indicates the D-Penicillamine released in a controlled manner. The in vitro release kinetics was assessed by Korsmeyer-Peppas equation and the ' $n$ ' value lies in between 0.557-0.693 indicates Non-Fickian diffusion process.

Conclusion: The results suggest that the developed KA intercalated microbeads are good potential drug carrier for the controlled release of D-PA.

Keywords: Sodium alginate (SA), Gelatin (GE), Kaolin (KA), Drug delivery, D-Penicillamine (D-PA)

(C) 2019 The Authors. Published by Innovare Academic Sciences Pvt Ltd. This is an open access article under the CC BY license (http://creativecommons.org/licenses/by/4.0/) DOI: http://dx.doi.org/10.22159/ijap.2019v11i5.34254

\section{INTRODUCTION}

In the last two decades, researchers have shown much interest in the use of nanoclay materials, most notably Kaolinite $[1,2]$, montmorillonite [3-5], diatomic [6-8], palygorskite [9], and halloysite [10-12], especially in biomedical field. This interest is largely stems from the human interest due to its biocompatibility, biostability, degradability, non-toxic and increase the drug efficiency in drug delivery systems [13].

Kaolin, $\mathrm{Al}_{2} \mathrm{Si}_{2} \mathrm{O}_{5}(\mathrm{OH})_{4} \quad(\mathrm{KA})$ is a two-dimensional (2D) natural aluminosilicate mineral which has been used in the field of biomedical applications for centuries [14]. In fact their medicinal utilities have been discovered by many traditional civilizations (Egyptians, Assyrians, Babylonians, Indians, Chinese), Greeks, Romans and medieval Arab Muslims till the recent times [15]. Kaolinite is used in the treatment of colitis, enteritis, dysentery and diarrhoea [16]. In the last few decades, researchers have paid huge attention to use KA minerals, because it can acts as active excipient in solid and semisolid pharmaceutical dosage forms and control the efficiency of the dosage forms which can improve the drug bioavailability [17-19].

Sodium Alginate (SA) is an anionic biodegradable natural polymer with 1,4- $\beta$-d-mannuronic acid (M block) and $\alpha$-l-guluronic acid (Gblock) residues [20]. The polymer forms a three-dimensional hydrogel which network due to the interaction between the carboxylic acid group (Gblock) and divalent counter ion (e. $\mathrm{g} \mathrm{Ca}^{2+}, \mathrm{Mg}^{2+}$ and $\mathrm{Zn}^{2+}$ ) and makes egg-box structure, results a cross linked hydrogels is useful in controlled release of bioactive molecules [21, 22]. SA crosslinked calcium beads could protect the acid sensitive drugs from gastric juice and consequently released from the beads in the intestine [23]. F. Martínez-Gómez et al., [24] reported that sodium alginate/polyvinyl alcohol hydrogels may be appropriate for use in the controlled release of drugs in the intestinal tract.

Gelatin (GE) is a natural protein material obtained by hydrolytic degradation of naturally occurring tropocollagen rod (a triple helical structure) which is a fundamental molecular unit of collagen $[25,26]$. In the recent decades it has been used in food and pharmaceutical industries due to its non-toxic, anti-carcinogenic, biocompatible, biodegradable, and also attractive starting material as a drug carrier due to its unique gelling properties [27]. Phadke et al., [28] reported that it was somewhat difficult to prepare long-lasting drug delivery vehicle because at body temperature it was dissolved very rapidly, makes a burst release of drug. In order to overcome these problems many researchers have prepared GE networks by crosslinking (Glutaraldehyde, formaldehyde and genipin), grafting and coating with other polymers. A. P. Rokhade et al., [29] reported that gelatin and NaCMC semi IPN microspheres for the effective encapsulation and controlled release of ketorolac tromethamine. Pal et al., [30] reported that gelatin-g-PAA nanoparticles are fairly suitable and function well as swelling controlled drug delivery system.

D-Penicillamine (D-PA) (fig. 1) is a biologically active aminothiol [31], which is used in the treatment of Wilson's disease and remove the excess of copper from the body, may be in the form of deep purple colour complex with D-PA by chelation process [32]. D-P is a potentially an effective chelator for some transition metals, hence it is used as a good choice for treatment of heavy metal poising [33].

A few articles have been reported the combination of SA and GE for controlled drug delivery [34-37], but as per best of our knowledge, the use of SA/GE/KA microbeads for controlled release of D-PA has not yet been investigated. In this research paper KA intercalated SA/GE microbeads were prepared. The developed beads were characterized by FTIR, SEM, EDS and X-RD. The dissolution experiments were performed to study the drug release characteristics of the microbeads and the results are presented here.

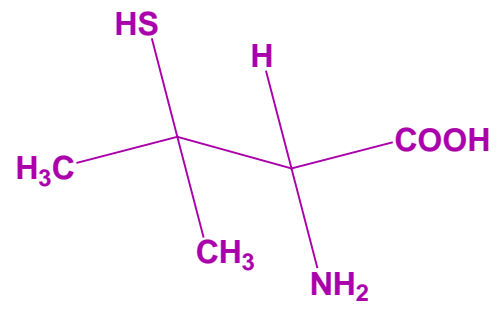

Fig. 1: Structure of D-penicillamine 


\section{MATERIALS AND METsHODS}

\section{Materials}

Gelatin and kaolin were purchased from Sigma-Aldrich (USA). Sodium alginate, Calcium chloride and Glutaraldehyde (GA) were purchased from Sd. Fine chemicals, Mumbai, India. D-Penicillamine a gift sample from Suven Life Sciences Ltd., Hyderabad. Water used was of high purity grade after double distillation.

\section{Preparation procedure}

\section{Preparation of SA/GE microbeads}

D-PA loaded microbeads were prepared by simple ionotropic gelation method [25]. Briefly, 3\% aqueous dispersion of SA and GE were prepared separately using double distilled water. Solutions of GE and SA were combined in different ratios (table 1). To this drug solution was added and stirred for $2 \mathrm{hr}$ by using a magnetic stirrer to get homogeneous solution. The above mixed solution was transferred drop wise into $\mathrm{CaCl}_{2}$ solution, the spherical beads formed instantly were kept for 5 min-and then transferred into $2 \%$ glutaraldehyde solution for crosslinking for $2 \mathrm{~h}$. The obtained wet beads were collected by decantation, washed three times with distilled water to remove the drug attached on the bead surface, and finally were dried in air overnight and then vacuum-dried to constant weight at $40^{\circ} \mathrm{C}$

\section{Preparation of SA/GE-kaolin nanoclay microbeads}

SA/GE blend solution was prepared by using above procedure. To this solution drug and nano kaolin clay was added in different ratios (table 1) and stirred for $2 \mathrm{hr}$ using magnetic stirrer. This solution was placed in sonication for $30 \mathrm{~min}$ to get homogeneous solution. Then the solution was transferred drop wise into $\mathrm{CaCl}_{2}$ solution, the spherical microbeads formed instantly were kept for $5 \mathrm{~min}$ and then transferred into $2 \%$ glutaraldehyde solution for crosslinking for $2 \mathrm{~h}$. The obtained wet beads were collected by decantation, washed three times with distilled water to remove the drug attached on the bead surface, and finally were dried in air overnight and then vacuum-dried to constant weight at $40^{\circ} \mathrm{C}$.

Table 1: Formulation, composition and \% Encapsulation Efficiency (\%EE) of all samples

\begin{tabular}{|c|c|c|c|c|c|c|}
\hline Formulation & SA (\% w/w) & GE $(\% w / w)$ & GA (\%) & Kaolin (mg) & Drug (mg) & $\% \mathrm{EE} \pm \mathrm{SD}$ \\
\hline F1 & 50 & 50 & 2 & 0 & 50 & $52.1 \pm 1.6$ \\
\hline $\mathrm{F} 2$ & 50 & 50 & 2 & 0 & 100 & $54.8 \pm 1.9$ \\
\hline F3 & 50 & 50 & 2 & 0 & 150 & $55.1 \pm 2.3$ \\
\hline F4 & 70 & 30 & 2 & 0 & 100 & $60.3 \pm 1.5$ \\
\hline F5 & 90 & 10 & 2 & 0 & 100 & $64.7 \pm 2.0$ \\
\hline F6 & 90 & 10 & 4.0 & 0 & 100 & $62.3 \pm 1.8$ \\
\hline F7 & 90 & 10 & 6.0 & 0 & 100 & $60.9 \pm 1.5$ \\
\hline F8 & 90 & 10 & 2 & 800 & 150 & $76.3 \pm 1.9$ \\
\hline F9 & 90 & 10 & 2 & 600 & 150 & $74.1 \pm 1.3$ \\
\hline F10 & 90 & 10 & 2 & 400 & 150 & $71.3 \pm 1.6$ \\
\hline F11 & 90 & 10 & 2 & 200 & 00 & 000 \\
\hline F12 & 90 & 10 & 2 & 0 & 00 & 000 \\
\hline
\end{tabular}

SD: Standard Deviation for $\mathrm{n}=3$

\section{Characterizations methods}

\section{Intercalation kinetics}

To determine the optimal time required for maximum intercalation of $D$ PA with KA. A known amount of D-PA (30 mg) and KA (100 mg) were mixed into $30 \mathrm{ml}$ of double distilled water with continuous stirring at 37 ${ }^{\circ} \mathrm{C}$. At regular intervals of time $(0.5,1,2,4,8$ and $14 \mathrm{~h})$ the reaction mixture was filtered and concentration of D-PA was analyzed using UV spectrophotometer at fixed $\lambda$-max value of $215.2 \mathrm{~nm}$.

\section{Effect of $\mathbf{p H}$}

The experiments were performed to estimate the most favourable $\mathrm{pH}$ for the intercalation of D-PA with KA. For this purpose, a known amount of D-PA (30 mg) and KA (100 mg) were dissolved into $30 \mathrm{ml}$ of different $\mathrm{pH}$ solutions $(2.0,4.6,6.0,7.4,8.5$ and 10.5$)$ and stirred at 300rpm for $1 \mathrm{~h}$ at $37^{\circ} \mathrm{C}$. The solution was then filtered and concentration of D-PA in the filtrate was analyzed by ultraviolet (UV) spectrophotometer (LabIndia, Mumbai, India) at the $\lambda$-max of $215.2 \mathrm{~nm}$.

\section{Fourier transform infrared (FTIR) spectral analysis}

FTIR (Bomem MB-3000 Make: Canada) with Horizon MBTM FTIR software was used to record the spectrum of pure SA, pure GE, pristine drug, placebo SA/GE microbeads, drug loaded SA/GE microbeads and drug loaded SA/GE/KA microbeads to find out the possible chemical interactions between polymer matrix, kaolin and drug. The finely powdered and dried samples $(5 \mathrm{mg})$ were mixed with $100 \mathrm{mg}$ of $\mathrm{KBr}$ in a pestle and mortar. Pellets were prepared under a hydraulic pressure of $600 \mathrm{~kg} / \mathrm{cm}^{2}$. These pellets were again crushed and repelleted. This step was repeated 2-3 times to get better reproducibility. Spectra were taken in the wavelength range $400-4000 \mathrm{~cm}^{-1}$.

\section{XG-ray diffraction (XRD) analysis}

The XG-ray diffraction of the of pristine drug, SA/GE placebo micro beads, drug loaded SA/GE micro beads, SA/GE/KA placebo micro beads and drug loaded SA/GE/KA micro beads were performed by a wide angle XG-ray scattering diffractometer (Panalytical XG-ray Diffractometer, model-X'pert Pro) with $\mathrm{CuK} \alpha$ radiation $(\lambda=1.54060)$ at a scanning rate of $5 \% \mathrm{~min}$ to determine the crystallinity.

\section{Field emission scanning electron microscopy (FESEM) analysis}

The morphological characterization of the microbeads was studied by using FESEM (Make: JEOL, Singapore. Model: JEOL JSM-7100F) with an accelerating voltage of $20 \mathrm{kV}$ equipped with an EDAX detector.

\section{Determination of encapsulation efficiency}

Percentage of encapsulation efficiency was estimated according to the formula and method reported in previous literature [38]. A known mass of beads $(10 \mathrm{mg})$ were immersed into $100 \mathrm{ml}$ of phosphate buffer solution ( $\mathrm{pH} 7.4$ containing 5\% absolute ethyl alcohol) for $24 \mathrm{~h}$ and then crushed the beads to ensure the complete extraction of D-PA from the beads. The absorbance of the clear supernatant buffer solution containing the extracted amount of D-PA was measured by UV-Vis spectrophotometer (LabIndia, Mumbai, India) at the $\lambda$-max value of $215.2 \mathrm{~nm}$ for D-PA with $\mathrm{pH} 7.4$ buffer solution as a blank. Concentration of drug was determined by using calibration curve constructed by series of D-PA standard solutions. Percentage of encapsulation efficiency was calculated by the following equations.

$$
\begin{gathered}
\text { Drug loading }(\%)=\frac{\text { Weight of drug in microbeads }}{\text { weight of microbeads }} \times 100 \\
\text { EE }(\%)=\frac{\text { Actual drug loading }}{\text { Theortical drug loading }} \times 100
\end{gathered}
$$

\section{In vitro drug release studies}

To study the in vitro drug release kinetics of different formulations were performed at $37{ }^{\circ} \mathrm{C}$ using a dissolution tester (Lab India, Mumbai, India) containing of eight baskets. Accurate quantity of dried beads $(100 \mathrm{mg}$ ) was immersed into $900 \mathrm{ml}$ of phosphate 
buffer solution pH7.4 at $50 \mathrm{rpm}$ to replicate intestinal fluid atmosphere. At a predetermined time intervals $5 \mathrm{ml}$ of sample withdrawn and $5 \mathrm{ml}$ of fresh Phosphate buffer solution (PBS) was added back to basket to keep total volume constant throughout the experiment. Amount of D-PA released was assayed by using a UVVis spectrophotometer at $215.2 \mathrm{~nm}$, and the released drug amount was obtained by using concentration versus absorbance calibration curve.

\section{Drug release kinetics}

The drug release kinetics was analyzed by fitting the data in to kinetic models, which include zeroth, first order, Higuchi and Korsmeyer-peppas [39-42]. Based on the goodness of data fit, the highest correlation coefficient was indicative of best-fit model to describe drug release kinetics [43].

\section{RESULTS AND DISCUSSION}

\section{Intercalation kinetics}

Intercalation kinetics reveals that the time required for intercalation of D-PA with KA by a rapid ion-exchange process between sodium ions of KA and cations of D-PA molecules. From fig. 2 it was clear that $14.9 \%$ of D-PA was intercalated in interlayer of KA within 90 $\mathrm{min}$, and remains constant up to $14 \mathrm{~h}$. Therefore we should keep 90 min time for interaction between D-PA and KA to avoid partial interaction in the following experiments.

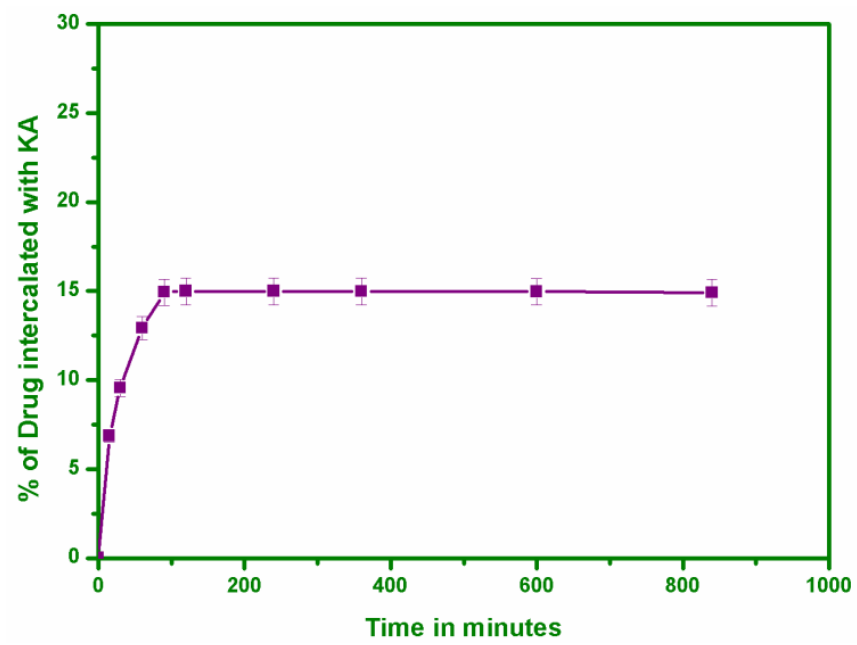

Fig. 2: Effect of time for Intercalation of D-PA with KA

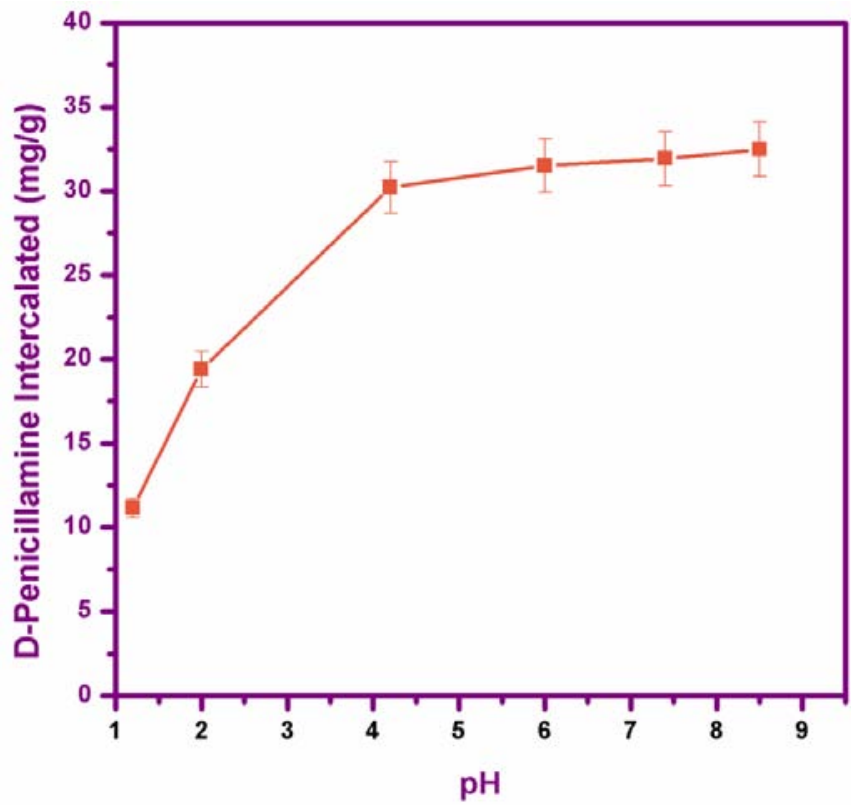

Fig. 3: Effect of pH on intercalation of D-PA into KA

\section{Effect of $\mathbf{p H}$}

The effect of $\mathrm{pH}$ on the intercalation of D-PA into the interlayer of KA is displayed in fig. 3. The results reveal that suitable $\mathrm{pH}$ range for intercalation of D-PA with KA is 6.0-8.5, because at this range intercalation of D-PA almost linear. At pH below 6.0 there was a decrease in intercalation of D-PA in KA layer. At a low $\mathrm{pH}$, hydronium ions increase, therefore a competition was arise between drug molecules and $\mathrm{H}^{+}$ions of $\mathrm{KA}$ layer, thus the system is flocculated; hence, adsorption decreases [44].

\section{Fourier transform infrared (FTIR) spectral analysis}

FTIR spectra of sodium alginate (a), gelatin (b), placebo SA/GE microbeads (c), pure drug (d) and drug loaded SA/GE microbeads are displayed in fig. 4. FTIR spectra of SA (fig. 4. a) shows a characteristic 
peaks at $3415 \mathrm{~cm}^{-1}$ corresponds to $\mathrm{O}-\mathrm{H}$ stretching frequency, the peaks arises at $1386 \mathrm{~cm}^{-1}$ and $1594 \mathrm{~cm}^{-1}$ assigned to asymmetric and symmetric stretching frequency of carboxylate group C-0. FTIR spectra of GE (fig. 4 . b) a broad band at $3341 \mathrm{~cm}^{-1}$ corresponded to stretching vibration of $\mathrm{N}-\mathrm{H}$ group, whereas amide $(\mathrm{C}=0)$ stretching vibrations are observed at $1616 \mathrm{~cm}^{-1}$. Further, the absorption band at $1373 \mathrm{~cm}^{-1}$ assigned to stretching vibrations of $\mathrm{C}-\mathrm{N}$ bond. FTIR spectra of placebo microbeads (fig. 4. c) a new band formed at $1612 \mathrm{~cm}^{-1}$ is assigned to $\mathrm{C}=\mathrm{N}$ stretching vibrations which indicates the interaction between-OH group of $\mathrm{SA}$ and $\mathrm{NH}_{2}$ group of GE which confirms the formation of microbeads [36]. The FTIR spectra of pristine D-PA (fig. 4. d) shows a band at 3473,2607 and $1627 \mathrm{~cm}^{-1}$ were due to-N-H,-S-H and-COOH stretching vibrations. Comparing the FTIR spectra of placebo microbeads and drug loaded microbeads, in placebo microbeads (fig. 4. c) a band appears at $1612 \mathrm{~cm}^{-1}$, whereas this band was shifted to lower wavelength i.e. $1596 \mathrm{~cm}^{-1}$ in drug loaded microbeads (fig. 4. e), which indicates the interaction occurs between polymer and drug molecule. The band at $2358 \mathrm{~cm}^{-1}$ further indicates the presence of S-H stretching frequency, which confirms the drug present in the drug loaded microbeads [38].

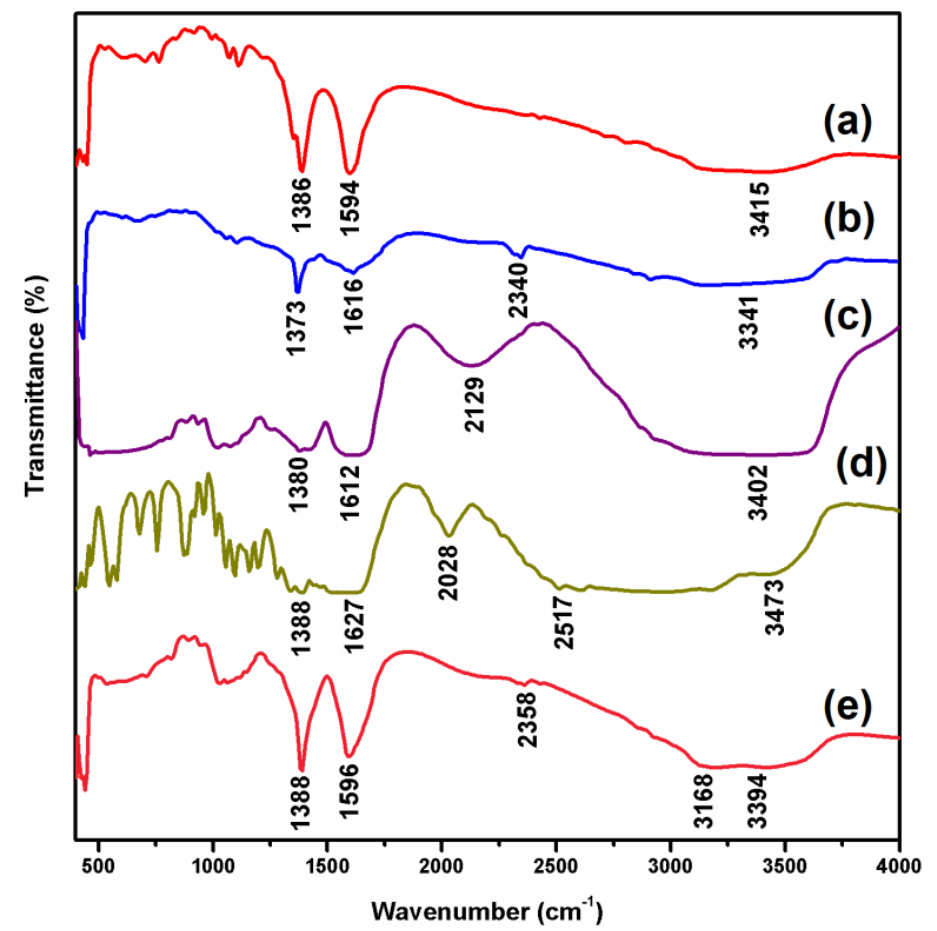

Fig. 4: FTIR spectra of sodium alginate (a), gelatin (b), placebo SA//GE microbeads (c), pure D-PA (d) and drug loaded SA/GE microbeads (e)

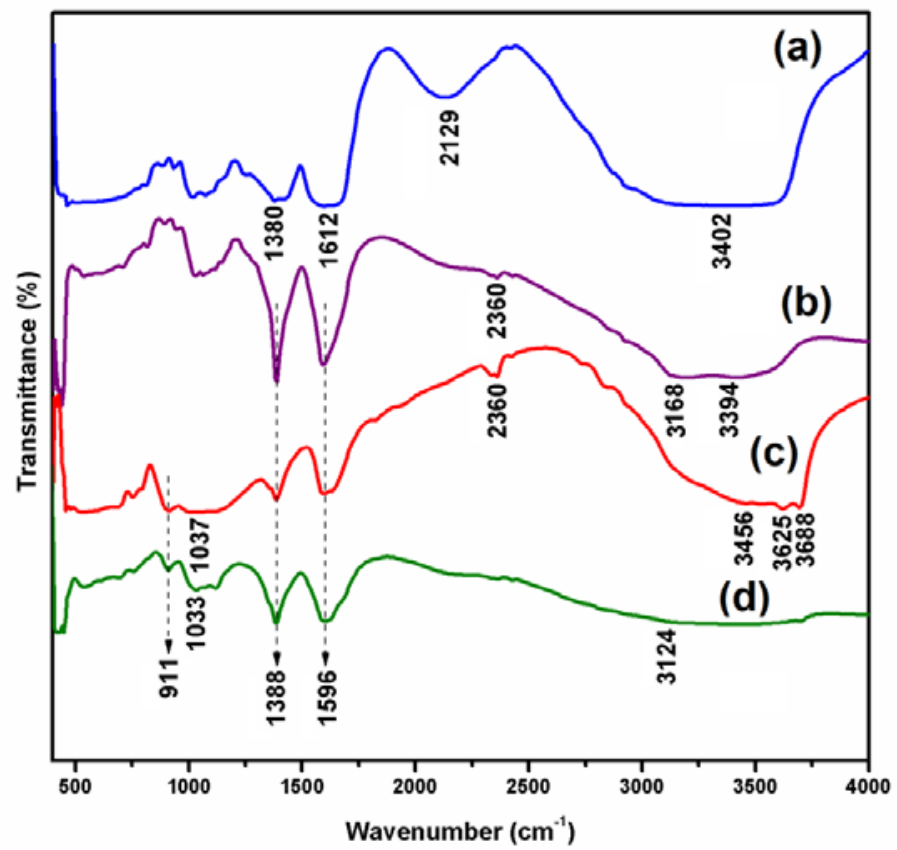

Fig. 5: FTIR spectra of SA/GE placebo microbeads (a), drug loaded SA/GE microbeads (b), Pure Kaolin (c) and drug loaded SA/GE/KA microbeads (d) 


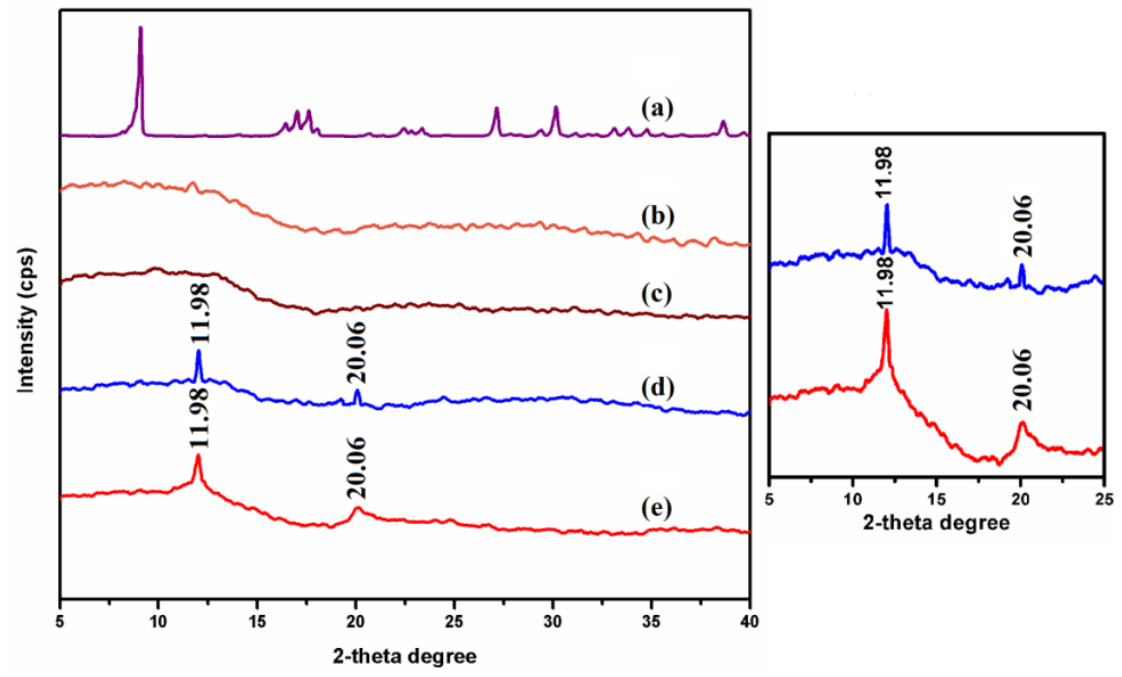

Fig. 6: Powder XRD patterns of pristine D-PA (a), SA/GE placebo micro beads (b), drug loaded SA/GE micro beads (c), SA/GE/KA placebo micro beads (d) and drug loaded SA/GE/KA micro beads (e)
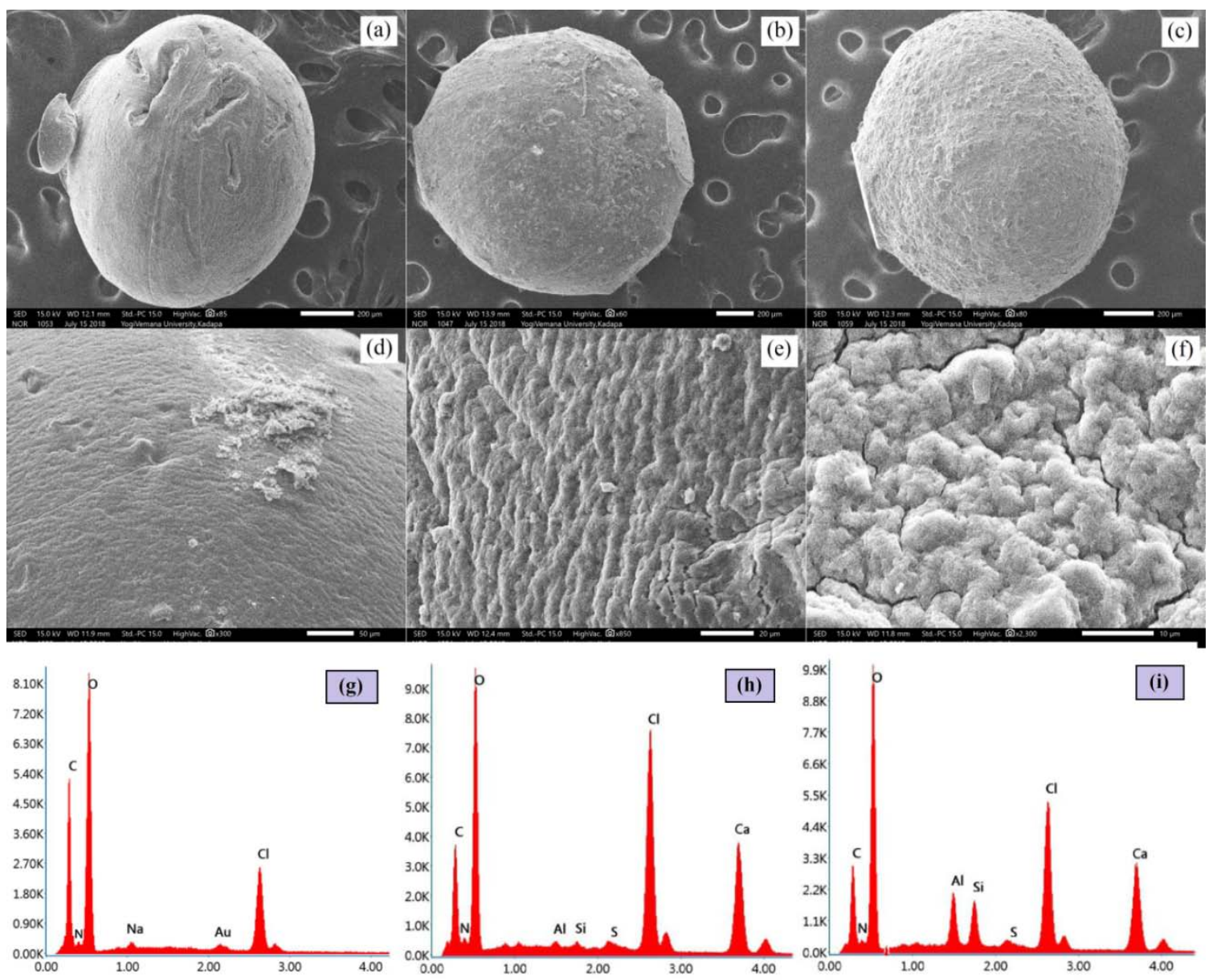

Fig. 7: FESEM images of SA/GE placebo micro beads (a,d), drug loaded SA/GE micro beads (b,e) and drug loaded SA/GE/KA micro beads $(\mathrm{c}, \mathrm{f})$ and EDS analysis of SA/GE placebo micro beads (g), drug loaded SA/GE micro beads (h) and drug loaded SA/GE/KA micro beads (i)

The FTIR spectrum of KA (fig. 5. c) shows distinct peaks at 3688 $\mathrm{cm}^{-1}$ and $3625 \mathrm{~cm}^{-1}(\mathrm{O}-\mathrm{H}$ stretching frequency of Si-OH and Al$\mathrm{Al}-\mathrm{OH}), 3456 \mathrm{~cm}^{-1}(\mathrm{H}-\mathrm{O}-\mathrm{H}$ stretching frequency of interlayer water), 1126 and $902 \mathrm{~cm}^{-1}$ corresponds to (Si-O-Si) stretching band. Comparing the FTIR spectra of KA and drug loaded $\mathrm{SA} / \mathrm{GE} / \mathrm{KA}$ microbeads (fig. 5. d), the intensity of KA peaks in drug loaded SA/GE/KA microbeads were weakened and new characteristic peaks appeared in drug loaded SA/GE/KA microbeads, this is due to intercalation between KA and active sites of polymer matrices and drug molecules. This confirms the KA intercalates with active sites of polymer matrices and drug molecules [13]. 


\section{X-ray diffraction (XRD) analysis}

Powder XRD patterns of pristine drug (a), SA/GE placebo micro beads (b), drug loaded SA/GE micro beads (c), SA/GE/KA placebo micro beads (d) and drug loaded SA/GE/KA micro beads (e) were displayed in fig. 8. The XRD pattern of D-PA (fig. 6. a) shows a characteristic peaks at $2 \theta$ of $9.10^{\circ}, 17.04^{\circ}, 17.64^{\circ}$, and $30.18^{\circ}$, represents the crystalline nature. Whereas the crystalline peaks are not completely observed in drug loaded SA/GE micro beads (fig. 6 . c), this suggests that the drug is dispersed at molecular level in the polymer matrix. The XRD patterns of SA/GE/KA placebo micro beads (fig. 6. d) shows a characteristic peaks at $11.98^{\circ}$ and $20.06^{\circ}$, indicates $\mathrm{KA}$ is present in the microbeads. Whereas these peaks were observed in the drug loaded SA/GE/KA micro beads (fig. 6. e) but the basal space of these peaks were increased this indicates the $\mathrm{KA}$ intercalates with the drug molecules.

\section{FESEM and EDS analysis}

In order to examine the surface morphology and chemical composition of KA loaded SA/PEG microbeads, surface SEM image and EDS analysis were performed. Fig. 7 presents the topographical images of placebo SA/GE microbeads (fig. 7. a and d), drug loaded SA/GE microbeads (fig. 7. $b$ and e) and drug loaded SA/GE/KA microbeads (fig. 7. $c$ and f). The SEM image of placebo SA/GE microbeads shows spherical, uniform and smooth surface with detectable pores. Whereas the drug loaded SA/GE microbeads shows pores on the surface this indicates drug should be loaded in the beads. The SEM image of drug loaded SA/GE/KA microbeads shows higher roughness compared with the drug loaded microbeads this is due to the hydrophilic character of clay which facilitates the miscibility of KA with SA/PEG blend matrix. Higher the KA contents in the polymer matrix higher the surface roughness in the microbeads. This is due to polymer matrix molecules that migrate toward the negative charge of the clay and entered into the clay layers and finally form a porous structure. This confirms the kaolin loaded in the microbeads [45].

The energy-dispersive X-ray spectra (EDS) analysis shows the presence of C, N, O elements in the placebo SA/GE microbeads (fig. 7. g) and drug loaded SA/GE/KA microbeads (fig. 7. h). On comparing the EDS spectra of placebo SA/GE microbeads and drug loaded SA/GE/KA microbeads in both spectra nitrogen peaks were observed indicating the presence of gelatin polymer. But the nitrogen peak in drug loaded SA/GE/KA microbeads is more intense than the peak present in placebo SA/GE microbeads and also additional Sulphur peak was observed in drug loaded SA/GE microbeads, which confirms the drug present in drug loaded SA/GE microbeads. Whereas the additional signals corresponding to the $\mathrm{Si}$ and $\mathrm{Al}$ elements were detected in the drug SA/GE/KA microbeads (fig. 7. i). This confirms the KA present in the microbeads.

\section{Determination of \% encapsulation efficiency (\%EE)}

The percentages of encapsulation efficiency (\%EE) of D-PA loaded microbeads are displayed in table 1 . The values are (\%EE) lies in between $52.1 \%$ to $76.3 \%$. This indicates the \%EE dependence on formulation parameters which include percentage of blend composition, concentration of GA and KA. The percentage of EE in formulations of F1, F2 and F3 decreases with the increase of gelatin composition in the blend composition, the decrease of encapsulation is due to gelatin might have assisted the diffusion of drug particles into the external surface to form pores in the matrix. As the concentration of glutaraldehyde increases in the formulations of F5, F6 and F7 the \%EE decreases, this is due to the crosslinking density increases the microbeads will become more rigid, thereby the crosslinker reduced the sizes of voids in the matrix. The formulations F8,F9,F10 with the increasing the amount of KA in the polymer matrix, the percentage of D-PA encapsulation efficiency increased this is due to kaolin has large specific area in its structure, good absorption capacity and develops hydrogen bonding between D-PA and hydroxyl groups present on surface of KA.

\section{In vitro drug release studies}

Based on the results of effect of $\mathrm{pH}$ on intercalation of clay and drug, the cumulative percentage of drug release was studied for all the formulations in $\mathrm{pH} 7.4$ buffer solution at $37^{\circ} \mathrm{C}$. In vitro drug release studies were discussed in terms of effect of polymer, effect of kaolin clay, drug variation and crosslinker variation.

\section{Effect of polymer variation}

The effect of polymer blend content was studied with constant loading of D-PA and glutaraldehyde. The drug release profiles of F2, F4 and F5 are $80.4 \%, 87 \%$ and $92 \%$ respectively are displayed in fig. 8 . This suggests that drug release rate increases with increasing content of sodium alginate, this is due to hydrophilic nature of sodium alginate [46], whereas the gelatin concentration increases the drug release rate decreases, this is due to the rigid nature of SA/GE blend component and the possible hydrogen-bonding interactions between - $\mathrm{COO}-$ of sodium alginate and $\mathrm{N}-\mathrm{H}$ group of gelatin.

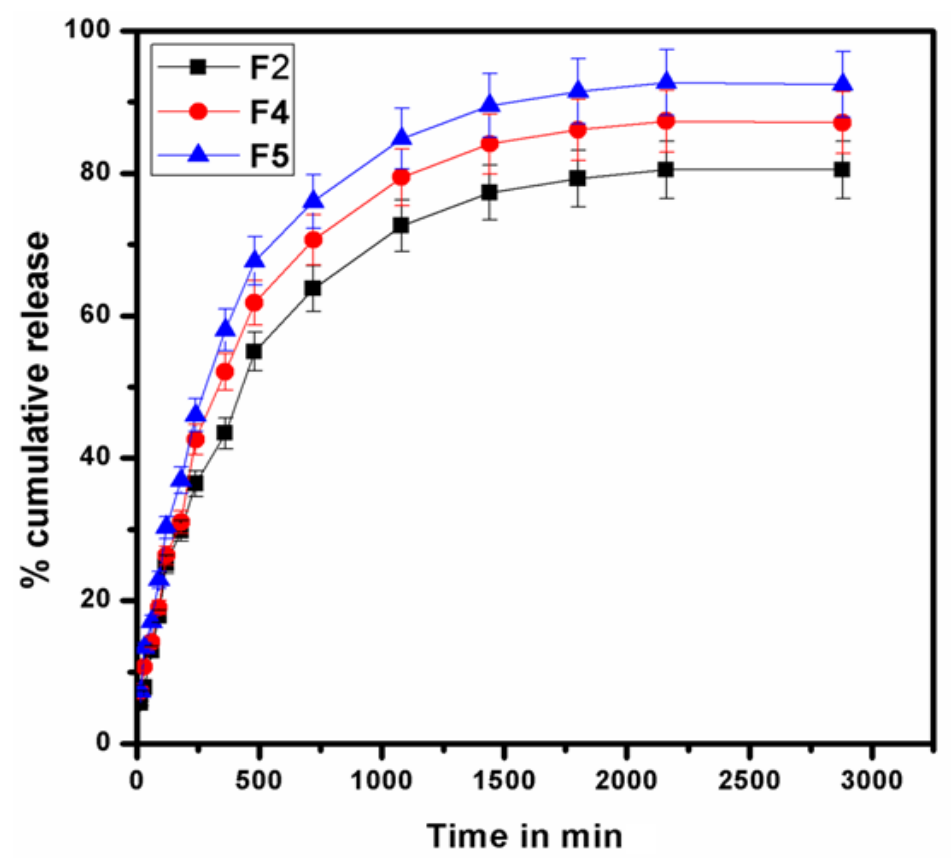

Fig. 8: Effect of polymer variation on in vitro release profiles for formulations F2 (50:50 ratioof SA: GE), F4 (70:30 ratio of SA: GE) and F5 (90:10 ratio of $S A: G E)$ at $\mathrm{pH7.4}$ 


\section{Effect of crosslinking agent}

To investigate the effect of crosslinker on in vitro release profiles was studied by plotting the cumulative $\%$ of drug release data against time for varying concentration of GA (i.e., 2.0, 4.0 and $6.0 \mathrm{ml}$ ) with constant of D-PA and polymer blend matrix are displayed in fig.
9. The drug release rate was higher when the microbeads containing small concentration of GA (i.e., $2 \mathrm{ml}$ ), whereas the release rate was lower when GA concentration was increased (i.e., $6 \mathrm{ml}$ ) in the polymer matrix. This is due to increased stiffness in polymer chains and reduced porosity of microbeads at higher crosslink densities, thereby hindering the transport of D-PA through the microbeads.

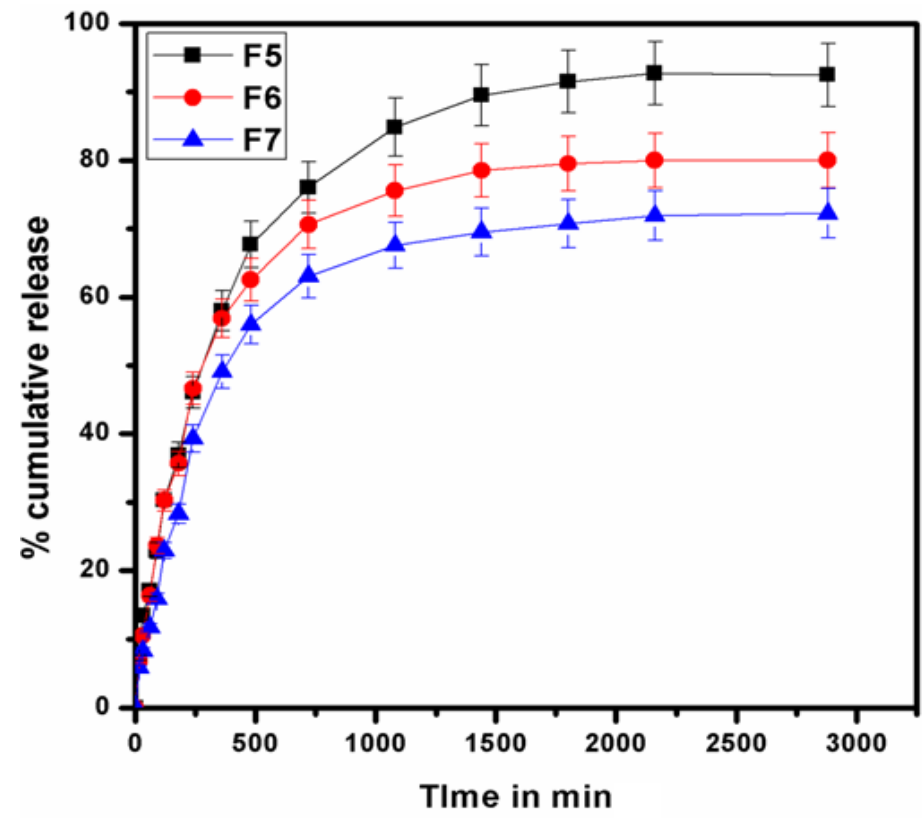

Fig. 9: Effect of crosslinker on in vitro release profiles for formulations F5 (2 ml), F6 (4 ml) and F7 (6 ml) at pH7.4

\section{Effect of drug loading}

Fig. 10 shows the effect of drug variation on in vitro release profiles was studied at a constant amount of polymer blend composition and crosslinker. The amount of drug loaded in the formulations F1, F2 and $\mathrm{F} 3$ are 50,100 and $150 \mathrm{mg}$ respectively. The \% of cumulative drug release for $\mathrm{F} 1, \mathrm{~F} 2$ and $\mathrm{F} 3$ are $77 \%, 80 \%$ and $83 \%$ respectively. The release profile shows a burst release with in $2 \mathrm{~h}$ and extended the release over the remaining time is due to swelling of the hydrophilic matrix. From the cumulative release data, it was found that the formulation which contains more amount of drug (F3) showed higher release rates and the formulation which contains lower amount of drug (F1) showed lower release rate. This indicates that release rates vary depending on the amount of drug in the matrices, i.e., the release rate was found to be significantly faster at higher amounts of drug this is due to osmotic pressure is high in higher concentration of drug which leads to quick diffusion of drug from the matrix to dissolution media [47]. The release rate was slower in F1, this might be due to the availability of extra free void spaces through which fewer drug molecules will transport [48].

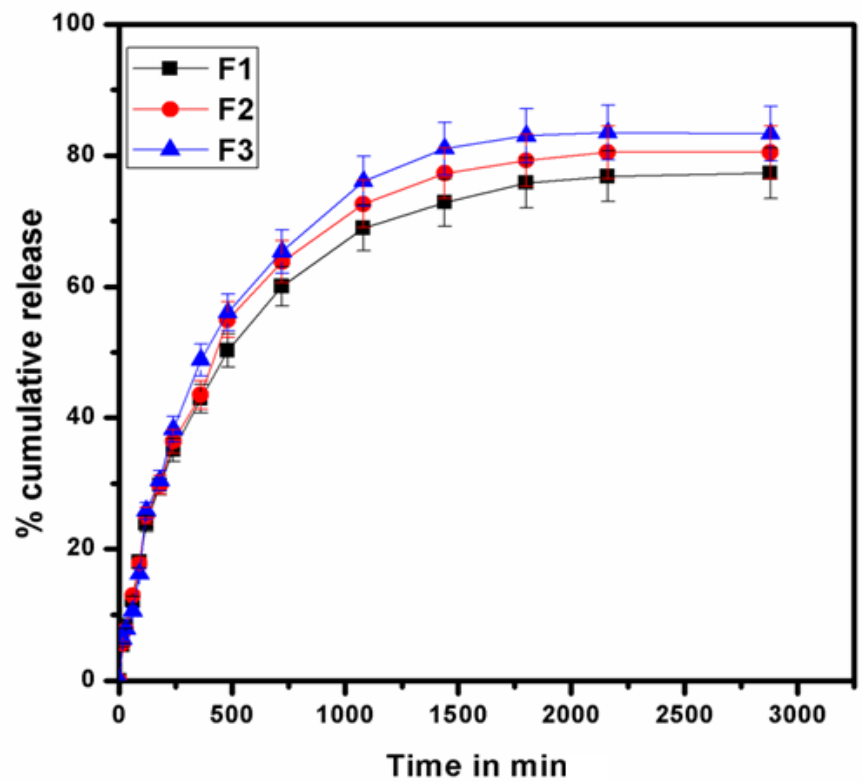

Fig. 10: Effect of \% drug loading on in vitro release profiles for formulations F1 (50 mg), F2 (100 mg), and F3 (150 mg) 


\section{Effect of kaolin clay}

To understand the effect of KA on in vitro release profiles was studied by plot the cumulative $\%$ of drug release data against time for varying amounts of KA (i.e., 400, $600 \mathrm{and} 800 \mathrm{mg}$ ) with constant D-PA and polymer blend matrix are displayed in fig. 11. The cumulative release rate of D-PA from microbeads containing KA is decreases as the concentration of KA increases, this is due to intercalated drug cannot be exchanged completely in ion exchange process with phosphate ions of the buffer solution and also the electrostatic attraction between amino groups of D-PA and anionic groups of KA layer which leads to incomplete release process.

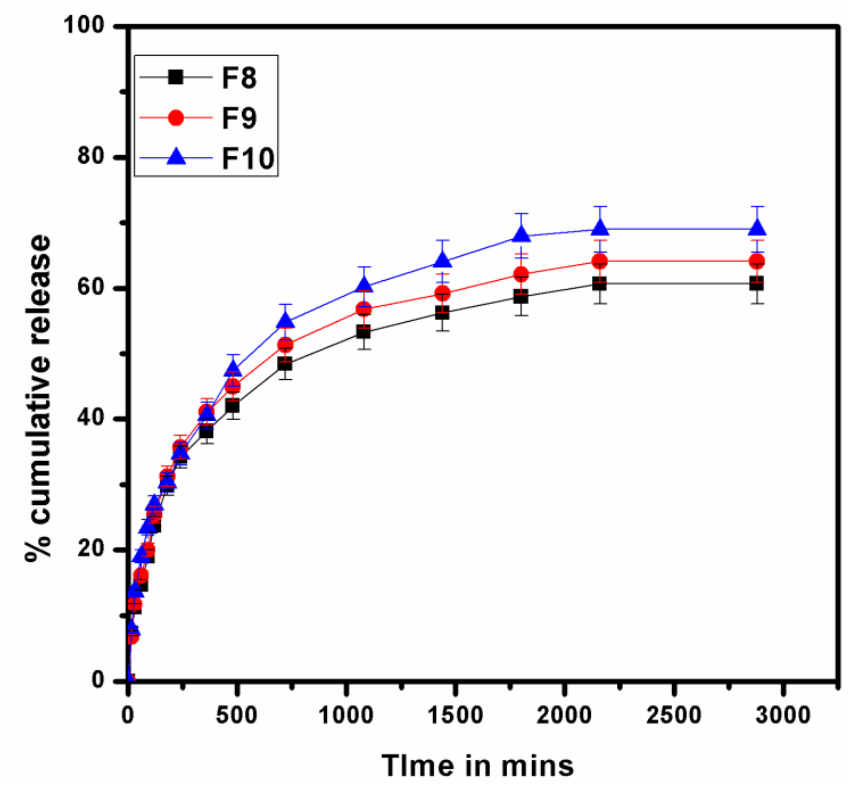

Fig. 11: Effect of kaolin nano clay variation on in vitro release profiles for formulations F8 (800 mg), F9 (600 mg) and F10 (400 mg)

\section{Drug release kinetics}

To find out the drug release mechanism, the data obtained from in vitro drug release studies in PBS (7.4) were fitted into different kinetic models including zero order, first order, Higuchi and Korsmeyer-Peppas models which are empirical in nature, the results of rate constant and correlation coefficient $\left(\mathrm{r}^{2}\right)$ of all formulations are displayed in table 2 . The correlation coefficient values indicated that drug release kinetics neither follow zero order nor first order. The $\mathrm{r}^{2}$ values were close to Higuchi and Korsmeyer peppas models. Therefore the drug release kinetics follows Higuchi model. According to Higuchi model the drug release from the microbeads, involves the penetration of liquid into the matrix and dissolves the drug, which then diffuses the drug into the exterior liquid through pores or intestinal channels. According to the principles of Higuchi and Korsmeyer-Peppas models it was clear that it the drug release process involves the simple diffusion phenomenon. Further, the type of diffusion was revealed by Korsmeyer-Peppas model. The first $60 \%$ drug release data were fitted into Korsmeyer-Peppas model.

$$
\frac{\mathrm{M}_{\mathrm{t}}}{\mathrm{M}_{\alpha}}=\mathrm{k} \mathrm{t}^{\mathrm{n}}
$$

Where, $\mathrm{Mt} / \mathrm{M} \alpha$ represents the fractional drug release at time $\mathrm{t}, \mathrm{k}$ is a constant characteristic of the drug-polymer system and $n$ is the release exponent indicating the type of drug release mechanism. The values $\mathrm{n}$ are obtained in the range of 0.557-0.693 indicating that the drug release mechanism followed Non-Fickian type of diffusion process. These results, along with correlation coefficients ' $r$ ' presented in Table.2. A similar observation was reported by $\mathrm{E}$. Yilmaz et al., [49] from the Preparation and characterization of $\mathrm{pH}$ sensitive semi-interpenetrating network hybrid hydrogels with sodium humate and kaolin.

Table 2: Drug release rate constant and correlation coefficient of all formulations after fitting drug release data into different mathematical models

\begin{tabular}{|c|c|c|c|c|c|c|c|c|c|}
\hline \multirow[t]{2}{*}{ S. No. } & \multirow[t]{2}{*}{ Sample } & \multicolumn{2}{|c|}{ Zero order } & \multicolumn{2}{|c|}{ First order } & \multicolumn{2}{|c|}{ Higuchi } & \multicolumn{2}{|c|}{ Korsmeyer-peppas } \\
\hline & & $\mathbf{k}_{0}$ & $\mathbf{r}^{2}$ & $\mathbf{k}_{1}$ & $\mathbf{r}^{2}$ & $\mathbf{k}_{\mathbf{H}}$ & $\mathbf{r}^{2}$ & $\mathbf{n}$ & $\mathbf{r}^{2}$ \\
\hline 1 & F1 & 8.246 & 0.934 & 1.971 & 0.974 & 20.59 & 0.988 & 0.693 & 0.988 \\
\hline 2 & $\mathrm{~F} 2$ & 7.847 & 0.957 & 1.975 & 0.985 & 20.64 & 0.992 & 0.679 & 0.990 \\
\hline 3 & F3 & 6.455 & 0.966 & 1.984 & 0.991 & 21.96 & 0.984 & 0.688 & 0.971 \\
\hline 4 & F4 & 8.566 & 0.982 & 1.977 & 0.993 & 22.57 & 0.988 & 0.587 & 0.978 \\
\hline 5 & F5 & 10.88 & 0.969 & 1.971 & 0.986 & 24.07 & 0.990 & 0.557 & 0.985 \\
\hline 6 & F6 & 10.21 & 0.950 & 1.972 & 0.990 & 24.18 & 0.979 & 0.675 & 0.994 \\
\hline 7 & F7 & 6.627 & 0.965 & 1.983 & 0.989 & 21.42 & 0.998 & 0.690 & 0.984 \\
\hline 8 & F8 & 5.918 & 0.972 & 1.983 & 0.992 & 20.51 & 0.990 & 0.671 & 0.976 \\
\hline 9 & F9 & 5.656 & 0.956 & 1.980 & 0.978 & 16.31 & 0.969 & 0.671 & 0.978 \\
\hline 10 & F10 & 6.803 & 0.967 & 1.979 & 0.991 & 20.56 & 0.992 & 0.661 & 0.979 \\
\hline
\end{tabular}

\section{CONCLUSION}

A simple ionotropic gelation method was used to fabricate D-PA loaded SA/GE/KA microbeads. Microbeads formation was confirmed by FTIR spectroscopy. X-RD reveals that the KA should be intercalated with the drug and also it confirms the molecular level dispersion of D-PA into microbeads. Scanning Electron Microscopy (SEM) studies reveal that the beads were in spherical shape with 
some wrinkled depressions on the surface. The in vitro release study indicates the D-PA released in a controlled manner. The in vitro release kinetics was assessed by different empirical equations and the data supported a Non-Fickian diffusion mechanism. Based on the results, Kaolin intercalated microbeads are promising material for drug carrier in emerging drug delivery systems.

\section{ACKNOWLEDGEMENT}

Research support provided by the department of Chemistry, S. K. University, Ananthapuramu, Andhra Pradesh, India.

\section{FINANCIAL SUPPORT AND SPONSORSHIP}

Two of the authors K. Chowdoji Rao and C. Madhavi acknowledge UGC-BSR financial support.

\section{AUTHORS CONTRIBUTIONS}

All the author have contributed equally

\section{CONFLICT OF INTERESTS}

The authors have indicated that they have no conflicts of interest regarding the content of this article

\section{REFERENCES}

1. Isabel Carretero M, Manuel Pozo. Clay and non-clay minerals in the pharmaceutical industry part I. Excipients and medical applications. Appl Clay Sci 2009;46:73-80.

2. Jeffrey G Lundin, Christopher L, McGann, Grant C Daniels, Benjamin C Streifel, James H Wynne. Hemostatic kaolinpolyurethane foam composites for multifunctional wound dressing applications. Materials Sci Eng C 2017;79:702-9.

3. Maide Gokce Bekaroglu, Fuad Nurili, Sevimİsci. Montmorillonite as imaging and drug delivery agent for cancer therapy. Appl Clay Sci 2018;162:469-77.

4. Shilpa Jain, Monika Datta. Montmorillonite-alginate microspheres as a delivery vehicle for oral extended release of venlafaxine hydrochloride. J Drug Delivery Sci Technol 2016;33:149-56.

5. Shuibo Hua, Huixia Yang, Aiqin Wang. A pH-sensitive nanocomposite microsphere based on chitosan and montmorillonite with in vitro reduction of the burst release effect chitosan/montmorillonite nanocomposite microspheres. Drug Development Industrial Pharm 2010;36:1106-14.

6. Janicijevic J, Krajisnik D, Calija B, Vasiljevic BN, Dobricic V, Dakovic A, et al. Modified local diatomite as potential functional drug carrier-a model study for diclofenac sodium. Int J Pharm 2015;496:466-74.

7. Ruggiero I, Terracciano M, Martucci NM, De Stefano L, Migliaccio N, Tate R, et al. Diatomite silica nanoparticles for drug delivery. Nanoscale Res Lett 2014;9:329-35.

8. Janicijevic J, Krajisnik D, Calija B, Dobricic V, Dakovic A, Krstic J, et al. Inorganically modified diatomite as a potential prolongedrelease drug carrier. Materials Sci Eng C 2014;42:412-20.

9. Jie Wu, Shijie Ding, Jing Chen, Suqin Zhou, Hongyan Ding. Preparation and drug release properties of chitosan/organomodified palygorskite microspheres. Int J Biol Macromol 2014;68:107-12.

10. Joanna Kurczewska, Michał Cegłowski, Beata Messyasz, Grzegorz Schroeder. Dendrimer-functionalized halloysite nanotubes for effective drug delivery. Appl Clay Sci 2018;153:134-43.

11. Yuri Lvov, Wencai Wang, Liqun Zhang, Rawil Fakhrullin. Halloysite clay nanotubes for loading and sustained release of functional compounds. Adv Mater 2016;28:1227-50.

12. Yuri M Lvov, Melgardt M DeVilliers, Rawil F Fakhrullin. The application of halloysite tubule nanoclay in drug delivery. Expert Opinion Drug Delivery 2016;13:977-86.

13. Yi Zhang, Mei Long, Peng Huang, Huaming Yang, Shi Chang, Yuehua $\mathrm{Hu}$, et al. Intercalated 2D nanoclay for emerging drug delivery in cancer therapy. Nano Res 2017;10:2633-43.

14. Yuping Liang, Congcong Xu, Guofeng Li, Tianchi Liu, Jun F Liang, Xing Wang. Graphene-kaolin composite sponge for rapid and riskless hemostasis. Colloids Surf B 2018;169:168-75.
15. Mahmoud E Awad, Alberto Lopez Galindo, Massimo Setti, Mahmoud M El-Rahmany, Cesar Viseras Iborra. Kaolinite in pharmaceutics and biomedicine. Int J Pharma 2017;533:34-48.

16. Omaimah M N Al Gohary. In vitro adsorption of mebeverine hydrochloride onto kaolin and its relationship to pharmacological effects of the drug in vivo. Pharma Acta Helvetiae 1997;72:11-21.

17. Myung Hun Kim, Goeun Choi, Ahmed Elzatahry, Ajayan Vinu, Young Bin Choy, Jin-Ho Choy. Review of clay-drug hybrid materials for biomedical applications: administration routes. Clays Clay Minerals 2016;64:115-30.

18. Lynda B Williams. Geomimicry: harnessing the antibacterial action of clays. Clay Minerals 2017;52:1-24.

19. Ghadiri M, Chrzanowskiab W, Rohanizadeh. Biomedical applications of cationic clay minerals. RSC Adv 2015;5:29467-81.

20. Mallikarjuna Reddy K, Ramesh Babu V, Krishna Rao KSV, Subha MCS, Chowdoji Rao K, Sairam M, et al. Temperature sensitive semi-IPN microspheres from sodium alginate and $\mathrm{n}$ isopropylacrylamide for controlled release of 5-fluorouracil. J Appl Polymer Sci 2008;107:2820-9.

21. Wu T, Huang J, Jiang Y, Hu Y, Ye X, Liu D, et al. Formation of hydrogels based on chitosan/alginate for the delivery of lysozyme and their antibacterial activity. Food Chem 2017;240:361-9.

22. Raghavendra V Kulkarni, Sreedhar V, Srinivas Mutalik, Mallikarjun Setty C, Biswanath Sa. Interpenetrating network hydrogel membranes of sodium alginate and poly(vinyl alcohol) for controlled release of prazosin hydrochloride through skin. Int J Biol Macromolecules 2010;47:520-7.

23. KV Ramana Reddy, MV Nagabhushanam. Process and parameters affecting drug release performance of prepared cross-linked alginate hydrogel beads for ezetimibe. Int J Pharm Pharm Sci 2016;9:254-62.

24. Fabian Martinez Gomez, Juan Guerrero, Betty Matsuhiro, Jorge Pavez. In vitro release of metformin hydrochloride from sodiumalginate/polyvinyl alcohol hydrogels. Carbohydrate Polymers 2017;155:182-91.

25. Madhusudana Rao K, Krishna Rao KSV, Ramanjaneyulu G, Chang Sik Ha. Curcumin encapsulated $\mathrm{pH}$ sensitive gelatin based interpenetrating polymeric network nanogels for anticancer drug delivery. Int J Pharma 2015;478:788-95.

26. M Das, PR Suguna, K Prasad, JV Vijaylakshmi, M Renuka. Extraction and characterization of gelatin: a functional biopolymer. Int J Pharm Pharm Sci 2017;9:239-42.

27. Van Den Bulcke AI, Bogdanov B, De Rooze N, Schacht EH, Cornelissen M, Berghmans H. Structural and rheological properties of methacrylamide modified gelatin hydrogels. Biomacromolecules 2000;1:31-8.

28. Keerti V Phadke, Lata S Manjeshwar, Tejraj M Aminabhavi. Microspheres of gelatin and poly(ethylene glycol) coated with ethyl cellulose for controlled release of metronidazole. Indian Eng Chem Res 2014;53:6575-84.

29. Ajit P Rokhade, Sunil A Agnihotri, Sangamesh A Patil, Nadagouda N Mallikarjuna, Padmakar V Kulkarni, Tejraj M Aminabhavi. Semiinterpenetrating polymer network microspheres of gelatin and sodium carboxymethyl cellulose for controlled release of ketorolac tromethamine. Carbohydrate Polymers 2006;65:243-52.

30. Pal A, Bajpai J, Bajpai AK. Poly (acrylic acid) grafted gelatin nanocarriers as swelling controlled drug delivery system for optimized release of paclitaxel from modified gelatin. J Drug Delivery Sci Technol 2018;45:323-33.

31. Abhinav Agarwal, Surendra Prasad, Radhey M Naik. Inhibitory kinetic spectrophotometric method for the quantitative estimation of D-penicillamine at micro levels. Microchemical J 2016;128:181-6.

32. Taheri M, Ahour F, Keshipour S. Sensitive and selective determination of $\mathrm{Cu} 2 \mathrm{p}$ at D-penicillamine functionalized nanocellulose modified pencil graphite electrode. J Phys Chem Solids 2018;117:180-7.

33. Prabhakar MN, Sajankumarji Rao U, Kumara Babu P, Subha MCS, Chowdoji Rao K. Interpenetrating polymer network hydrogel membranes of PLA and SA for control release of penicillamine drug. Indian J Adv Chem Sci 2013;1:240-9. 
34. Sarika PR, Nirmala Rachel James. Polyelectrolyte complex nanoparticles from cationised gelatin and sodium alginate for curcumin delivery. Carbohydrate Polymers 2016;148:354-61.

35. Sarika PR, Nirmala Rachel James, Anil kumar PR, Deepa K Raj. Preparation, characterization and biological evaluation of curcumin loaded alginate aldehyde-gelatin nanogels. Materials Sci Eng C 2016;68:251-7.

36. Utkarsh Bhutani, Anindita Laha, Kishalay Mitra, Saptarshi Majumdar. Sodium alginate and gelatin hydrogels: viscosity effect on hydrophobic drug release. Materials Lett 2016;164:76-9.

37. Madhumathi K, Jeevana Rekha L, Sampath Kumar TS. Tailoring antibiotic release for the treatment of periodontal infrabony defects using bioactive gelatin-alginate/apatite nanocomposite films. J Drug Delivery Sci Technol 2018;43:57-64.

38. Madhusudana Rao K, Mallikarjuna B, Krishna Rao KSV, Prabhakar MN, Chowdoji Rao K, Subha MCS. Preparation and characterization of $\mathrm{pH}$ sensitive poly(vinyl alcohol)/sodium carboxymethyl cellulose IPN microspheres for in vitro release studies of an anti-cancer drug. Polym Bull 2012;68:1905-19.

39. Ikhuoria M Arhewoh, Augustine O Okhamafe. An overview of site-specific delivery of orally administered proteins/peptides and modelling considerations. J Med Biomed Res 2004;3:7-20.

40. Donbrow M, Samuelov Y. Zero order drug delivery from double-layered porous films: release rate profiles from ethyl cellulose, hydroxypropyl cellulose and polyethylene glycol mixtures. J Pharm Pharmacol 1980;32:463-70.

41. Suvakanta Dash, Padala Narasimha Murthy, Lilakanta Nath, Prasanta Chowdhury. Kinetic modeling on drug release from controlled drug delivery systems. Acta Poloniae Pharm Drug Res 2010;67:217-23.
42. Paulo Costa, Jose Manuel Sousa Lobo. Modeling and comparison of dissolution profiles. Eur J Pharm Sci 2001;13:123-33.

43. Korsmeyer RW, Gurny R, Doelker E, Buri P, Peppas NA. Mechanisms of solute release from porous hydrophilic polymers. Int J Pharm 1983;15:25-35.

44. Aleanizy FS, Alqahtani F, Al Gohary O, El Tahir E, Al Shalabi R. Determination and characterization of metronidazole-kaolin interaction. Saudi Pharma J 2015;23:167-76.

45. Sonia Bouzid Rekik, Sana Gassara, Jamel Bouaziz, Andre Deratani, Semia Baklouti. Development and characterization of porous membranes based onkaolin/chitosan composite. Appl Clay Sci 2017;143:1-9.

46. Madhavi C, Kumara Babu P, Maruthi Y, Parandhama A, Sreekanth Reddy 0, Chowdoji Rao K, et al. Sodium alginatelocust bean gum IPN hydrogel beads for the controlled delivery of nimesulide-anti-inflammatory drug. Int J Pharm Pharm Sci 2017;9:245-52.

47. Keerti V Phadke, Lata S Manjeshwar, Tejraj M Aminabhavi, MP Sathisha. Cellulose acetate butyrate bilayer coated microspheres for controlled release of ciprfloxacin. Polymer Bull 2018;75:1329-48.

48. Praveen B Kajjari, Lata S Manjeshwar, Tejraj M Aminabhavi. Semi-interpenetrating polymer network hydrogel blend microspheres of gelatin and hydroxyethyl cellulose for controlled release of theophylline. Indian Eng Chem Res 2011;50:7833-40.

49. Elif Yilmaz, Gulcihan Guzel Kaya, Huseyin Deveci. Preparation and characterization of $\mathrm{pH}$-sensitive semi-interpenetrating network hybrid hydrogels with sodium humate and kaolin. Appl Clay Sci 2018;162:311-6. 